VOL. $21(1980), 81-91$.

\title{
AN IMPLICIT FUNCTION THEOREM WITH SYMMETRIES AND ITS APPLICATION TO NONLINEAR EIGENVALUE ERUATIONS
}

\author{
E.N. DANCER
}

\begin{abstract}
In this paper we prove a $G$-invariant implicit function theorem and indicate how it can be used to improve an earlier result of the author on the bifurcation of solutions of nonlinear equations in the presence of continuous groups of symmetries. We also use our theorem to show that, under reasonable hypotheses, the method of looking for solutions in invariant subspaces yields all solutions. This can be used to answer a question raiser by Sattinger [J. Math. Phys. 19 (1978), 1729]. The abstract result is also of interest because it provides a theorem which should be of use in other symmetric situations.
\end{abstract}

We assume that $G$ is a compact lie group, $E$ is a finite-dimensional linear space with norm \|\| and $\left\{T_{g}\right\}_{g \in G}$ is a representation of $G$ in $B(E)$, the linear operators on $E$. Assume that $f: E \times R \rightarrow E$ is continuous, that the partial derivative $f_{1}^{\prime}(x, t)$ exists and is continuous and that $f$ is $T_{g}$-invariant. (In other words, $f\left(r_{g} x, t\right)=r_{g} f(x, t)$ for $g \in G, x \in E$ and $t \in R)$. In addition, suppose that $f(k, 0)=0$ and that the dimension of the kernel of the partial derivative $f_{1}^{\prime}(k, 0)$ is equal to the dimension of the manifold $M \equiv\left\{T_{g} k: g \in G\right\}$. (By the symetries, $f(m, 0)=0$ if $m \in M$. Hence $f_{1}^{\prime}(k, 0) h=0$ if $h \in T_{k}(M)$, the tangent space to $M$ at $k$. Thus we are assuming the Received 27 April 1979. 
kernel is as small as possible.) Finally, following the notation in [4], we assume that $M$ and $G$ have Property $P$, that is that

$$
\left\{h \in T_{k}(M): T_{g} h=h \text { for all } g \in G_{k}\right\}=\{0\},
$$

where $G_{k}=\left\{g \in G: T_{g} k=k\right\}$. Geometrically, this is the same as assuming that the only $G$-invariant vector field on $M$ is the trivial one. (This is proved in $\S 2$ of [4].) Note that we are abusing the notation slightly because we should really speak of the action of $G$ on $M$ having Property $P$.

THEOREM (G-invariant implicit function theorem). Under the above assumptions, there is a continuous function $w: M \times(-\varepsilon, \varepsilon) \rightarrow E$ such that $f(w(m, t), t)=0$ if $m \in M$ and $|t|<\varepsilon, w(m, 0)=m, w$ is $T_{g}^{-}$ invariant and, if $f(x, t)=0$ with $x$ near $M$ and $t$ near zero, then $x=w(m, t)$ for some $m \in M$.

REMARK. It follows easily from the proof that, for each $t$ near zero, the zeros of $f(x, t)=0$ near $M$ form a manifold diffeomorphic to $M$ (where, in fact, $w(, t)$ is the diffeomorphism).

Before proving the theorem, we prove two simple technical lemmas. The first is well-known but we give a proof for completeness.

LEMMA 1. Assume that $H$ is a finite-dimensional normed linear space, $S: H \rightarrow H$ is linear, $\left\{T_{g}\right\}_{g \in Y}$ is a continuous representation of a compact group $Y$ in $B(H)$ and $S$ is $T_{g}$-invariant. Then there exist projections $P$ and $\bar{P}$ onto $K(S)$ and $R(S)$ such that $P$ and $\vec{P}$ commute with $T_{g}$ for $g$ in $Y$. (Here $K(S)$ and $R(S)$ denote the kernel and range of $S$ respectively.)

Proof. Since $H$ is finite-dimensional, we may assume that $H$ is a Hilbert space. Then, by [3, Theorem 6.2.1], we may assume that the scalar product on $H$ is $T_{g}$-invariant. We then simply define $P$ and $\bar{P}$ to be orthogonal (with respect to this scalar product) projections on $K(S)$ and $R(S)$ respectively. It is easy to check (cf. $[4, \S 1]$ ) that $P$ and $\bar{P}$ have the required invariance.

REMARK. The result remains true if $H$ is a Banach space, $S \in B(H)$ (where $B(H)$ now denotes the bounded linear operators on $H$ ) and $S$ is 
Fredholm (in the notation of [5]). For example, if $P_{1}$ is a projection on $K(S)$, we simply define $P=\int_{g \in Y} T_{g^{-1}}^{P T} g d \mu(g)$, where $\mu$ is an invariant Haar measure on $Y$.

LEMMA 2. Assume that the conditions of Lemma 1 hold. Let $C=R(I-\bar{P})$. Then there exists a linear isomorphism $B: K(S) \rightarrow C$ which commutes with $T_{g}$ for $g$ in $Y$.

Proof. We use some elementary representation theory (as in [11]). By Lemma 1, $H=K(S) \oplus F$ where $F \equiv R(I-P)$ is $T_{g}$-invariant for $g$ in $Y$. Similarly, $H=R(S) \oplus C$ where $C$ is $T_{g}$-invariant for $g$ in $Y$. Now,

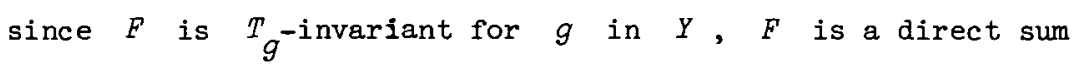
$F=\sum_{i=1}^{p} \oplus F_{i}$, where each $F_{i}$ is invariant and $\left\{T_{g}\right\}_{g \in Y}$ act irreducibly on each $F_{i}$. (In other word̄s, we are writing $F$ as a direct sum of irreducible representations.) Similarly $K(S)=\sum_{i=1}^{q} \oplus N_{i}$ where each $N_{i}$ is invariant and $Y$ acts irreducibly on each $N_{i}$. Hence

$$
E=K(S) \oplus F=\sum_{i=1}^{q} \oplus N_{i} \oplus \sum_{i=1}^{p} \oplus F_{i} \text {. }
$$

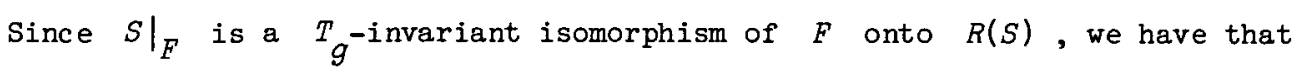
(up to isomorphism) $R(S)=\sum_{i=1}^{p} \oplus F_{i}$. Hence, if we write $C=\sum_{i=1}^{t} \oplus C_{i}$ where each $C_{i}$ is invariant and irreducible, we find that

$$
E=\sum_{i=1}^{t} \oplus c_{i} \oplus \sum_{i=1}^{p} \oplus F_{i}
$$

If a finite-dimensional representation is expressed as a direct sum of irreducible representations, then the number of times each irreducible representation occurs (that is its weight) is uniquely determined. Hence it follows from (1) and (2) that $\sum_{i=1}^{q} \oplus N_{i}$ and $\sum_{i=1}^{t} \oplus C_{i}$ are equivalent representations. Thus there is an invariant isomorphism of $K(S)$ and $C$. 
This proves Lemma 2.

Note that most books on representation theory discuss complex representations. However, the part of the theory that we require is true for real representations. Alternatively, Lemma 2 can be proved by using complexifications.

REMARK. Simple examples show that Lemma 2 may fail if $S$ is a bounded linear operator on a Banach space even if $S$ is Fredholm of index zero. However, it is true if $S$ is Fredholm of index zero and zero is an isolated point of the spectrum of $S$.

Proof of Theorem. First note that, by the tubular neighbourhood theorem ( $c f$. Bredon [3], Theorem 6.2.2), there is a G-invariant neighbourhood $U$ of $M$ in $E$ such that every point $x$ in $M$ can be uniquely expressed in the form $m+n$, where $m \in M$ and $n \in N_{m}(M)$. (Here $N_{m}(M)$ denotes the orthogonal complement of $T_{m}(M)$ in $E$ with respect to a $G$-invariant scalar product on $E$.) We look for solutions of $f(x, \lambda)=0$ of the form $x=m+u$, where $u \in N_{m}(M)$. (For the moment we keep $m$ fixed.) Consider the equation

$$
\bar{P}_{m} f(m+u, t)=0 \text {, }
$$

where $\bar{P}_{m}$ is a $G_{m}$-invariant projection onto $R\left(f_{1}^{\prime}(m, 0)\right)$. Note that

(i) $G_{m}$ denotes the isotropy group,

(ii) $f_{I}^{\prime}(m, 0)$ is $G_{m}$-invariant because $g$ is and because $m$ is fixed by $G_{m}$, and

(iii) there is such a projection by Lemma 1 . Since $f_{1}^{\prime}(m, 0)$ is an isomorphism of $N_{m}(M)$ onto $R\left(f_{1}^{\prime}(m, 0)\right)$, the implicit function theorem implies that there exist $\varepsilon_{m}>0$ and $\delta_{m}>0$ such that equation (3) has a unique solution $u=n(m, t)$ in $\left\{u \in N_{m}(M):\|u\|<\delta_{m}\right\}$ for each $t$ in $\left(-\delta_{m}, \delta_{m}\right)$. As in [4], we find from the group invariance that $\delta_{m}$ and $\varepsilon_{m}$ are independent of $m$ and that $n$ commutes with the $T_{g}$ 's for $g$ in $G$. There is one point to 
be noted here. To ensure that $\bar{P}_{m}$ 's commute with the $T_{g}$ 's, we find a suitable $\bar{P}_{k}$ and then we define $\bar{P}_{m}=T_{g} \bar{P}_{k}{ }_{g}{ }^{-1}$ where $T_{g}{ }^{k}=m$. It is easy to check that $\bar{P}_{m}$ is well-defined.) Hence the solutions of $f(x, t)=0$ near $M \times\{0\}$ are the solutions of

$$
\left(I-\bar{P}_{m}\right) f(m+n(m, t), t)=0 \text {. }
$$

Now, as we mentioned before, $f_{1}^{\prime}(k, 0)$ is a $G_{k}$-invariant linear mapping of $E$ into itself. Hence, by Lemma 2 , there is a $G_{k}$-invariant linear isomorphism $W_{k}$ of $R\left(I-\bar{P}_{k}\right)$ onto $k\left(f_{1}^{\prime}(k, 0)\right)=T_{k}(M)$. We define $W_{m}: R\left(I-\bar{P}_{m}\right) \rightarrow T_{m}(M)$ by the formula $W_{m}=T_{g} W_{k}{ }_{g}-I$, where $T_{g} k=m$. It is easy to see that $W_{m}$ is well-defined and is an isomorphism of $R\left(I-\vec{P}_{m}\right)$ onto $T_{m}(M)$. Thus equation (4) is equivalent to the equation

$$
s(m, t) \equiv W_{m}\left(I-\bar{P}_{m}\right) f(m+n(m, t), t)=0 \text {. }
$$

From its construction, $s(m, t) \in T_{m}(M)$ and a simple calculation shows that $T_{g} s(m, t)=s\left(T_{g} m, t\right)$. Thus $s$ is a $G$-invariant vector field on $M$. Since Property $P$ holds, $s \equiv 0$. Hence $(m+n(m, t), t)$ is a solution of $f(x, t)=0$. Since $n$ is $G$-invariant and since our proof shows these are the only solutions near $M \times\{0\}$, the result follows.

GENERAL REMARK. Most of the remarks in $\$ \S 1-3$ of [4] have analogues here.

REMARKS. 1. The remarks in $\$ 3$ of [4] concerning the necessity of Property $P$ still hold here. It is also possible to prove that our conditions on $f(x, 0)$ are the weakest for which we can expect both an existence and uniqueness result. As in $\$ 2$ of [4], Property $P$ places restrictions on the manner in which the group $G$ can act on $M$. For example, if Property $\mathrm{P}$ holds, then $G$ is not abelian (unless $M$ is discrete).

2. Our proof above is based on an idea in [4]. (A special case was considered there.) The proof there also assumes that $K\left(f_{1}^{\prime}(k, 0)\right) \cap R\left(f_{1}^{\prime}(k, 0)\right)=\{0\}$. Property $\mathrm{P}$ could be replaced by an equivalent condition on $R\left(I-\vec{P}_{k}\right)$ but Property $P$ has the great advantage 
that it is independent of $f$. It is also more convenient to work with tangent vectors.

3. One major use of the theorem is as follows. In applications, (for example, in [8], [9] and [10] where further references can be found), many authors have looked for solutions of $f(x, t)=0$ by choosing $k$ in $M$ and then looking for solutions near $k$ in the subspace $N^{k} \equiv\left\{x \in E: T_{g} x=x\right.$ for all $\left.g \in G_{k}\right\}$ by using the implicit function theorem. They then use the symmetries to generate orbits of solutions. Because $T_{g}\left(k+n_{k}(\lambda)\right)=k+n_{k}(\lambda)$ for $g$ in $G_{k}$, our theorem shows that, under natural hypotheses, this method yields all solutions. This result can easily be used to answer a question raised on p. 1729 of [8]. However, an example in $\$ 5$ of [4] shows that it is sometimes easier to apply our theorem directly rather than to use this method. There are two other cases where it can be proved that the method of looking for solutions in invariant subspaces yields all solutions near $M \times\{0\}$. We delete our assumption on the dimension of the kernel of $f_{1}^{\prime}(k, 0)$. The result is true if either

(i) $M$ is a principal orbit or, more generally,

(ii) $k\left(f_{1}^{\prime}(k, 0)\right) \cap N_{k}(M) \subseteq N^{k}$.

(One proves that, if $P_{k} f(k+n, t)=0,(n, t)$ is small and $n \in N_{k}(M)$, then $T_{g} n=n$ for all $g$ in $G_{k}$. Here $P_{k}$ is a suitable invariant projection.)

4. There is one case where Property $P$ can be deleted. Assume that there is a $G$-invariant scalar product $\{$,$\rangle and a map F: E \times R \rightarrow R$ such that $f$ is the gradient of $F$ (with respect to the first variable for the above scalar product). Property $P$ is unnecessary in this case because it turns out that, in this case, our vector field on $M$ is essentially the gradient of $F(m+n(m, t), t)$ and thus has a zero. (For technical reasons, it is convenient to use the method in [2].)

5. For simplicity, we assume that $f_{1}^{\prime}(k, 0)$ is self adjoint in this remark. It is natural to conjecture that we at least have existence of 
solutions if we replace our condition that $\operatorname{dim} k\left(f_{1}^{\prime}(k, 0)\right)=\operatorname{dim} M$ by the assumption that index $\left(\left.\left(I-P_{k}\right) f\left(k^{+}, 0\right)\right|_{N_{k}(M)}, 0\right)$ is defined and non-zero, where $P_{k}$ is the orthogonal projection onto $T_{k}(M)$. Unfortunately, this is false. There are examples where this holds but the equation $f(x, t)=0$ has no solutions near $M$ for every small non-zero $t$. However, as in $[4, \S 1]$, it is possible to prove the existence of solutions under weaker assumptions than those of the theorem. (The conditions in [4] can be greatly improved.)

6. Our methods can be used to obtain a similar theorem for a $G$-invariant mapping $f: E \times R \rightarrow E$, where $E$ and $F$ are two finitedimensional spaces of the same dimension, $\left\{T_{g}\right\}_{g \in G}$ is a representation of $G$ on $E$ and $\left\{\bar{T}_{g}\right\}_{g \in G}$ is a representation of $G$ on $F$. The one difference is that, except when the two representations are equivalent as $G_{k}$ representations, the theorem is only true with Property P replaced by the analogous condition on $R\left(I-\bar{P}_{k}\right)$. Note that the assumptions that $\operatorname{dim} M=\operatorname{dim} K\left(F_{1}^{\prime}(k, 0)\right)$ and that the analogue of Property $\mathrm{P}$ holds forces the representations to be $G_{k}$-equivalent in many cases. Moreover, in many problems, other assumptions imply that $f_{l}^{\prime}(0, t)$ is a $G$-invariant isomorphism of $E$ onto $F$ for some $t$ and hence the representations are $G$-equivalent.

7. Our result can also be generalized to the case where $E$ is a Banach space provided that $B(E)$ now denotes the bounded linear operators on $E$, we assume that the mapping $g \rightarrow T_{g}$ is smooth (as a mapping of $G$ into $B(E)$ ) and we assume that $f_{1}^{\prime}(k, 0)$ is Fredholm of index zero. We merely sketch this as the proof requires a little more care. Firstly, by a comment after Lemma 1 , there exist $G_{k}$-invariant projections $P_{k}$ and $\bar{P}_{k}$ onto $K\left(f_{1}^{\prime}(k, 0)\right)$ and $R\left(f_{1}^{\prime}(k, 0)\right)$ respectively. We then define a projection $P_{m}$ onto $K\left(f_{1}^{\prime}(m, 0)\right)$ by the formula $P_{m}=T_{g} P_{k} T_{g^{-1}}$ with ${ } g^{k}=m$. It can be shown that the mapping $m \rightarrow P_{m}$ is smooth. We replace $N_{m}(M)$ by $R\left(I-P_{m}\right)$. We use the implicit function theorem (as in the usual 
proof of the tubular neighbourhood theorem) to show that every $x$ near $M$ in $E$ can be uniquely expressed in the form $m+n$ where $n \in R\left(I-P_{m}\right)$ and $n$ is small. The rest of the proof proceeds as before.

The only other difference is that, as in Remark 6, we can only prove the result with Property $P$ replaced by the analogous condition on $R\left(I-\bar{P}_{k}\right)$. (However, if zero is an isolated point of the spectrum of $f_{1}^{\prime}(k, 0)$, the remark after the proof of Lemma 2 enables us to prove the analogue of our theorem.)

We now obtain a proposition which clarifies the role of Property $P$. For our purposes, the second part is probably the most important. The first part was pointed out to me by Dr M. Field.

PROPOSITION. (i) $M$ and $G$ have Property $P$ if and only if $\operatorname{dim} N\left(G_{k}\right)>\operatorname{dim} G_{k}$, where $N\left(G_{k}\right)$ denotes the normalizer of $G_{k}$.

(ii) If $k$ is an isolated solution of $f(x, 0)=0$ in $N^{k} \times\{0\}$, then $M$ and $G$ have Property $P$.

Proof. (i) We first prove that, if $\operatorname{dim} N\left(G_{k}\right)>\operatorname{dim} G_{k}$, then $M$ and $G$ do not have Property P. To see this, note that, if $\operatorname{dim} N\left(G_{k}\right)>\operatorname{dim} G_{k}$, then the same is true of the dimensions of their corresponding Lie algebras. Thus we can choose a one parameter subgroup $g(s) \equiv \exp s u$ of $N\left(G_{k}\right)$ with $u$ not in the Lie algebra of $G_{k}$. Since $g(s) \in N\left(G_{k}\right), T_{h} T_{g(s)} k=T_{g(s)} T_{h}, k=T_{g(s)^{k}}$ if $h \in G_{k}$ (where $\left.h^{\prime} \in G_{k}\right)$. Thus, by differentiating, $\left.v \equiv \frac{d}{d s} T_{g(s)} k\right|_{s=0}$ satisfies $T_{h} v=v$

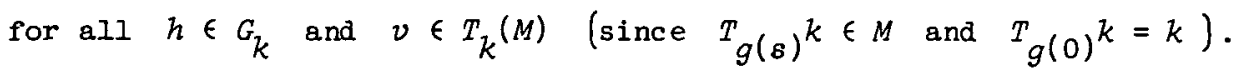
Moreover $v \neq 0$. (This follows by examining the arguments on pages 302-303 of [3].) Hence $v$ is non-zero, $v \in T_{k}(M)$ and $T_{h} v=v$ for all $h \in G_{k}$. Hence, by the discussion in $\S 2$ of [4], $M$ and $G$ do not have Property P.

Conversely, we prove that, if $M$ and $G$ do not have Property $\mathrm{P}$, then $\operatorname{dim} N\left(G_{k}\right)>\operatorname{dim} G_{k}$. Since $M$ and $G$ do not have Property $\mathrm{P}$, there is a $G$-invariant vector field $v$ on $M$ with no zeros. (This is proved in $\$ 2$ 
of [4].) Since $G$ acts transitively and smoothly on $M$, it is easy to show that $v$ is smooth. Consider the corresponding flow $F$ on $M$. ( $F$ is obtained by solving the differential equation $x^{\prime}(s)=v(x(s))$.) since $v$ is $G$-invariant, it is easy to show that $F$ is $G$-invariant. Since $T_{g} F(s, k)=F\left(s, T_{g} k\right)=F(s, k)$ if $g \in G_{k}$.

$$
F(s, k) \in\left\{x \in M: T_{g} x=x \text { for all } g \in G_{k}\right\} \text {. }
$$

Now it is easy to see that there is an $\varepsilon>0$ and a continuous function

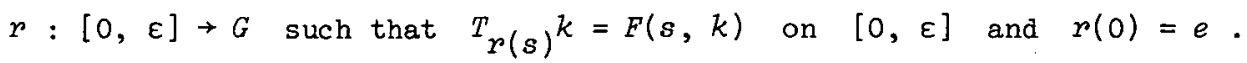
(This follows since fibre bundles have local cross-sections.) Now, if $h \in G_{k}$,

$$
\begin{aligned}
T_{(r(s))^{-1} h r(s)} k=\left(T_{r(s)}\right)^{-1} T_{h^{T}}{ }_{r(s)} & =\left(T_{r(s)}\right)^{-1} T_{h} F(s, k) \\
& =\left(T_{r(s)}\right)^{-1} F(s, k) \quad\left(\text { since } T_{h} k=k\right) \\
& \left.=\left(T_{r(s)}\right)^{-I_{T_{r}}}{ }^{k}\right)^{k} \\
& =k .
\end{aligned}
$$

Thus $r(s)^{-1} h r(s) \in G_{k}$. Hence $r(s)$ is a curve in $N\left(G_{k}\right)$. Since $F_{l}^{\prime}(0, k)=v(k) \neq 0, r(s)$ is not completely contained in $G_{k}$. Hence every neighbourhood of $e$ in $N\left(G_{k}\right)$ contains points not in $G_{k}$ and thus $\operatorname{dim} N\left(G_{k}\right)>\operatorname{dim} G_{k}$.

(ii) Suppose by way of contradiction that $M$ and $G$ do not have Property P. By the proof of part (i), there is a non-constant curve $F(s, k)$ contained in $N^{k} \cap M$ such that $F(0, k)=k$. (It is nonconstant because $v(k) \neq 0$.) Since each point in $M$ is a solution of $f(x, 0)=0$, it follows that $k$ is a non-isolated solution (in $N^{k}$ ) of $f(x, 0)=0$. Since this contradicts our assumption, we have completed the proof.

The results on normalizers in $\$ 3.9 .4$ of [1] can now be used to obtain a necessary condition for $M$ and $G$ to have Property $\mathrm{P}$ which is expressed in terms of the Lie algebra of $G$. If $G_{k}$ is connected, this condition 
is also sufficient.

Our theorem applies in particular to the bifurcation problem in [4]. There it was assumed that $E$ is a real. Banach space, $A: E \rightarrow E$ is Fréchet differentiable, $A(0)=0$ and there is a continuous representation $\left\{T_{g}\right\}_{g \in G}$ of $G$ in $B(E)$ such that $A$ is $T_{g}$-invariant. There, under appropriate hypotheses, it was shown that the study of the small solutions of $A(x)=\lambda x$ with $\lambda$ near $\lambda_{0}$ reduces to the study of the solutions near $\left(0, \lambda_{0}\right)$ of an equation

$$
\left(\lambda-\lambda_{0}\right) u=Q C(u)+\operatorname{Rem}(u, \lambda),
$$

where $u$ is in a finite-dimensional subspace $Y$ of $E, Q C$ is an $s$-homogeneous operator, Rem is a higher order term and each term commutes with the symmetries. For simplicity, we now only look for solutions with $\lambda \geq \lambda_{0}$. If $Q C(u) \neq 0$ for $u \in Y \backslash\{0\}$, it is shown in [4] that every solution of (6) with $\|u\|+\left|\lambda-\lambda_{0}\right|$ small, $u \neq 0$ and $\lambda \geq \lambda_{0}$ is of the form $\left(\left(\lambda-\lambda_{0}\right) / \tau\right)^{1 /(s-1)} y$, where $\tau>0$ and $y$ is near a solution $z$ of $Q C(x)=\tau x$ with $\|z\|=1$. Hence, by a change of variable $u=\left(\lambda-\lambda_{0}\right)^{1 /(s-1)} x$, we can reduce this problem to one where our theorem applies (if certain hypotheses hold). If we apply our theorem we find that Theorem 1 in [4] still holds if Assumption $H 6$ there is weakened to $\operatorname{dim} K\left(\tau I-Q C^{\prime}(k)\right)=\operatorname{dim} M$.

Our method of weakening Assumption H6 in Theorem 1 of [4] can also be used to correspondingly weaken Assumption $\mathrm{H} 6$ in Theorem 3 of [4]. More generally, the method in part (ii) of $\$ 4$ of [4] could be combined with the methods here to study the case where $f(, t)-f(, 0)$ is only invariant under a closed subgroup $G^{\prime}$ of $G$.

To complete this paper, we want to make a remark on a result in [4]. We follow the notation there. It can be proved that in Remark 1 at the end of $\S 1$, the assumption that $\left.T_{g}\right|_{K}=I$ for every $g \in G_{k}$ implies that index $\left(F_{k}, 0\right)= \pm$ index $\left(\left.F_{k}\right|_{F}, 0\right)$. Hence the first method there is contained in the second.

Added in Proof. Remark 5 can be improved to obtain a "best possible" result on the existence of solutions. 


\section{References}

[1] N. Bourbaki, Eléments de mathématique. Fascicule XXXVII: Groupes et algèbres de Lie. Chapitre II: Algèbres de Lie libres; Chapitre III: Groupes de Lie (Actualités Scientifiques et Industrielles, 1349. Hermann, Paris 1972).

[2] Reinhold Böhme, "Nichtlineare Störung der isolierten Eigenwerte selbstadjungierter Operatoren", Math. Z. 123 (1971), 61-92.

[3] Glen E. Bredon, Introduction to compact transformation groups (Pure and Applied Mathematics, 46. Academic Press, New York and London, 1972) .

[4] E.N. Dancer, "On the existence of bifurcating solutions in the presence of symmetries", Proc. Roy. Soc. Edinburgh (to appear).

[5] Seymour Boldberg, Unbounded linear operators (McGraw-Hill, New York, 1966).

[6] Werner Greub, Stephen Halperin, and Ray Vanstone, Connections, curvature, and cohomology. Volume I: De Rham cohomology of manifolds and vector bundles (Pure and Applied Mathematics, 47. Academic Press, New York and London, 1972).

[7] D.H. Sattinger, "Group representation theory, bifurcation theory and pattern formation", J. Funct. Anal. 28 (1978), 58-101.

[8] D.H. Sattinger, "Bifurcation from rotationally invariant states", $J$. Math. Phys. 19 (1978), 1720-1732.

[9] A. Stokes, "Invariant subspaces, symnetry and alternative problems", Bulz. Inst. Math. Acad. Sinica 3 (1975), no. 1, 7-14.

[10] A. Vanderbauwhede, "Alternative problems and invariant subspaces", $J$. Math. Anal. Appl. 63 (1978), 1-8.

[11] N.Ja. Vilenkin, Special functions and the theory of group representa representations (translated by V.N. Singh. Translations of Mathematical Monographs, 22. American Mathematical Society, Providence, Rhode Island, 1968).

Department of Mathematics, University of New England, Armidale, New South Wales 2351, Australia. 